\title{
Arecibo Survey of HI Emission from Disk Galaxies at Redshift $\mathrm{z} \sim 0.2$
}

\author{
B. Catinella ${ }^{1}$, M. P. Haynes ${ }^{2}$, J. P. Gardner ${ }^{3}$, A. J. Connolly ${ }^{3}$, \\ and R. Giovanelli ${ }^{2}$ \\ ${ }^{1}$ NAIC-Arecibo Observatory $\dagger$, Arecibo, PR 00612, USA; email: bcatinel@naic.edu \\ ${ }^{2}$ Center for Radiophysics and Space Research and NAIC, Cornell University, Ithaca, NY \\ 14853, USA \\ ${ }^{3}$ Dept. of Physics and Astronomy, University of Pittsburgh, Pittsburgh, PA 15260, USA
}

\begin{abstract}
We present results from a targeted survey undertaken with the 305m Arecibo radiotelescope to detect HI-line emission from disk galaxies at redshift $\mathrm{z}>0.16$. Among other applications, this dataset will be used to study the evolution of disk scaling relations at intermediate redshifts. Compared to optical velocity widths, Hi measurements sample a larger fraction of the disks, where the rotation curves are typically flat, and are not affected by slit smearing and misalignment or by aperture effects. Thus, in contrast to studies based on optical spectroscopy, this dataset allows for a direct comparison with the local Tully-Fisher relation that is technique independent.
\end{abstract}

Keywords. galaxies: kinematics and dynamics, radio lines: galaxies

\section{Evolution of disk scaling relations}

A very interesting and much debated issue is the evolution of the Tully-Fisher relation (TFR; Tully \& Fisher 1977) over cosmic time. If galaxies were more luminous in the past, we should observe an offset in the TFR derived independently at high and low redshift. However, studies based on optical spectroscopy (e.g., Flores et al. 2006) have reached conflicting conclusions. Results vary from substantial luminosity evolution (in excess of one magnitude with respect to the $\mathrm{z}=0 \mathrm{TFR}$ ) even at modest $\mathrm{z}$ to no significant change up to $\mathrm{z} \sim 1$. Evidence for evolution of the TFR or its lack remains inconclusive.

\section{Arecibo observations}

Detection of $21 \mathrm{~cm}$ emission from galaxies at $\mathrm{z}>0.1$ is difficult: the signals are weak, accurate redshifts of the targets must be known in advance, the presence of radio frequency interference restricts the redshift windows practically accessible, and the higher number of galaxies sampled by the antenna beam at higher redshifts leads to increased confusion problems. In fact, such observations have become feasible only recently thanks to technical improvements at Arecibo (Gregorian upgrade and installation of the new L-wide receiver, with access to frequencies down to $1.12 \mathrm{GHz}$ ) and to the availability of the Sloan Digital Sky Survey (SDSS; York et al. 2000), which provides high quality photometric images, accurate redshifts, and line emission information for $\sim 700,000$ galaxies.

The targets for Hi spectroscopy were extracted from the SDSS database based on their redshift, optical emission line strength, inclination, disk morphology, and relative

$\dagger$ The Arecibo Observatory is part of the National Astronomy and Ionosphere Center, which is operated by Cornell University under a cooperative agreement with the NSF. 

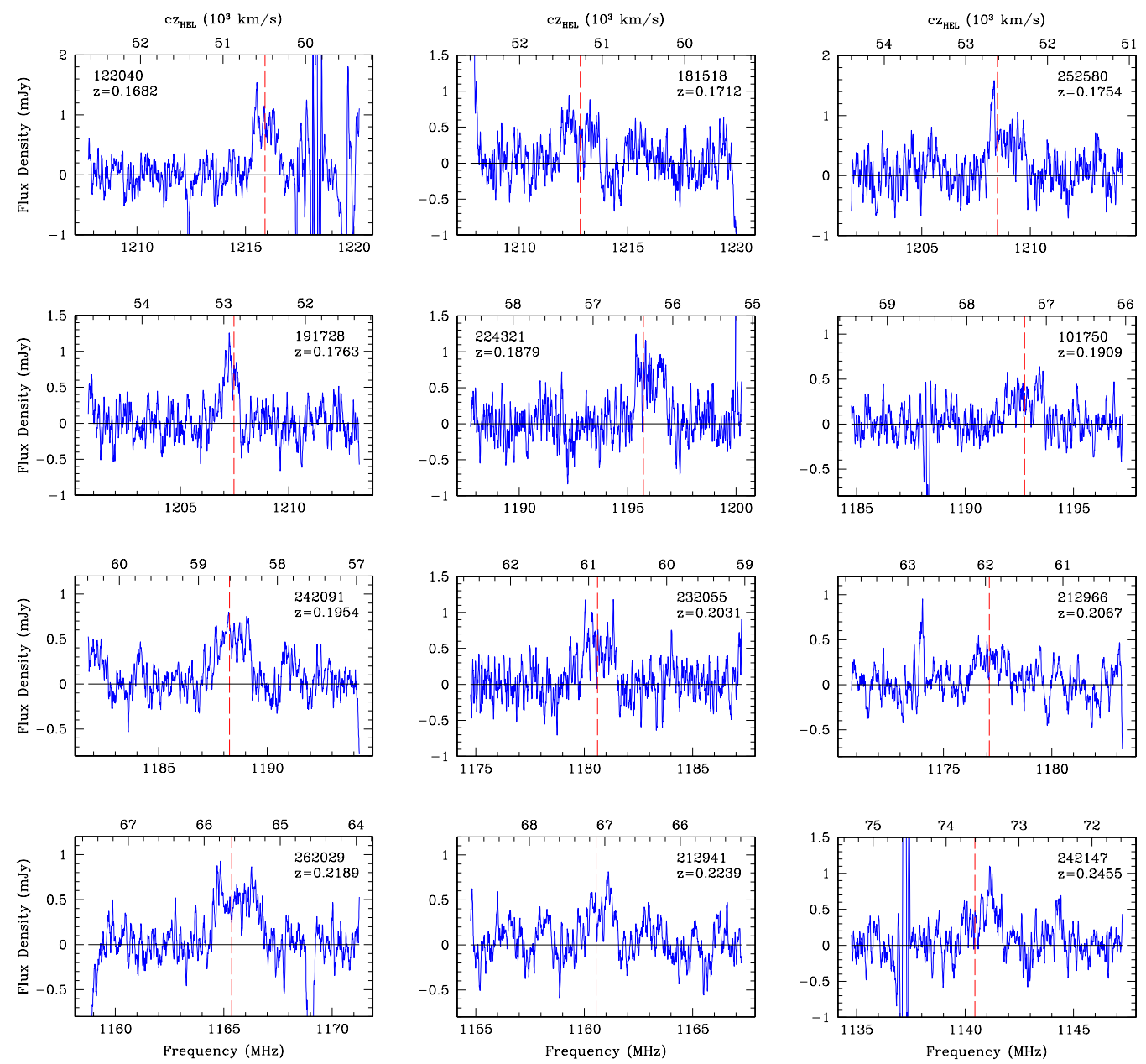

Figure 1. Calibrated, smoothed Hi spectra of 12 galaxies with $0.17 \leqslant z \leqslant 0.25$ detected at Arecibo. The red lines indicate the frequencies corresponding to the SDSS redshifts of the targets. Each spectrum is labeled with its AGC identifier and SDSS redshift.

isolation (to minimize confusion within the beam). Hi profiles of adequate quality for flux and velocity width measurement were obtained for $\sim 20$ galaxies in the redshift range $0.17-0.25$. The HI spectra of 12 of these detections are presented in Fig. 1 . The average total integration time varied between 2 and 6 hours, primarily depending on the redshift of the target. This sample includes the highest redshift detections of Hi emission from individual, normal galaxies to date.

The analysis of the selection biases of this sample, necessary to establish if this dataset is consistent with TFR evolution, is in progress and will be presented elsewhere.

\section{References}

Flores, H., et al. 2006, A\&\&A, 455, 107

Tully, R. B., \& Fisher, J. R. 1977, A\&A A, 54, 661

York, D. G., et al. 2000, AJ, 120, 1579 